\title{
Avaliação crítica dos efeitos adversos do tratamento anti-retroviral no feto, recém-nascido e lactente
}

\author{
Critical evaluation of the adverse effects of anti-retroviral treatment \\ on the fetus, the newborn and the infant \\ Heloisa Helena de Sousa Marques ${ }^{1}$
}

\section{RESUMO}

A introdução da profilaxia da transmissão vertical do HIV com o uso de drogas anti-retrovirais aliadas a outras medidas resultou na redução de crianças infectadas pelo HIV em nosso meio. Esses medicamentos são de uso recente e não há dados conclusivos para definir a sua segurança durante a gravidez. Esse artigo analisa, através de revisão bibliográfica, os possíveis efeitos dessas drogas sobre o feto, o recém-nascido e durante a infância, incluindo o potencial de teratogenicidade, carcinogênese e sua toxicidade. As descrições incluem a identificação de risco aumentado na criança para toxicidade mitocondrial, alterações neurológicas, hematológicas, hepáticas em crianças expostas ao HIV. Baseados nos conhecimentos atuais, considera-se que o benefício na acentuada redução da transmissão vertical do HIV com o uso de drogas anti-retrovirais ultrapassa os riscos eventuais dos efeitos adversos. Entretanto, até que experiência de longo prazo se acumule, é necessário acompanhamento clínico especializado das crianças expostas. Nessa revisão sugere-se uma rotina de acompanhamento clínico-laboratorial ao longo do primeiro ano de vida e depois anualmente até o final da adolescência.

PALAVRAS-CHAVE: Terapia anti-retroviral de alta atividade/efeitos adversos; Transmissão vertical de doença; Complicações infecciosas na gravidez; Infecções por HIV; Feto; Lactente; Recém-nascido

\section{ABSTRACT}

The implementation of prophylactic antiretroviral drugs and others strategies to prevent vertical HIV transmission in Brazil has reduced the number of newly HIV-infected children. Since these drugs are only recently available for treatment, there is no data of conclusive safety during pregnancy and fetus and newborn. This article reviews the possible effects of these drugs in fetus, newborn and childhood, including teratogenesis, carcinogenesis and toxicity. In the literature there are an increasing description of adverse effects such as mithocondrial toxicity, neurological symptoms, blood and liver toxicity in HIV-exposed not infected children. Antiretroviral treatment recommendations are based on principles that known benefit to the pregnat woman should be mantained unless adverse effects outweight the benefit. A long term follow up of HIV- exposed children is proposed to better understanding of these potential toxicity. In this review the author suggests a practical clinical and laboratory routinary evaluation during the first years of life until adolescence for HIV-exposed not infected children.

KEYWORDS: Antiretroviral therapy, highly active/adverse effects; Disease transmission, vertical; Pregnancy complications, infectious; HIV Infections; Fetus; Infant; Recém-nascido 
Introdução

O benefício da profilaxia da transmissão vertical do HIV (virus da imunodeficiência humana) com o uso de drogas anti-retrovirais aliadas a outras medidas é inquestionável. Atualmente, o risco de transmissão vertical com o uso de terapia potente e outras medidas varia desde 0 a $3 \%$, ao passo que com o uso isolado de zidovudina (AZT) situava-se ao redor de $8 \%$ e sem o uso de anti-retroviral era de $25 \%{ }^{1}$. Contudo, deve-se considerar que se trata de doença recente, para a qual se usam medicamentos novos, num momento especial de desenvolvimento - a gestação - quando a homeostase da mulher está adaptada para garantir a sua sobrevivência e o desenvolvimento de seu feto. Assim, o uso de medicamentos ao longo da gravidez deve ser criterioso, embasado na melhor evidência científica disponivel.

$\mathrm{Na}$ decisão sobre o uso de anti-retrovirais durante a gestação, aspectos como os efeitos das drogas sobre o feto e o recém-nascido, incluindo o potencial de teratogenicidade, carcinogênese, além da farmacocinética e toxicidade de drogas que ultrapassam a barreira transplacentária, devem ser considerados. O dano potencial depende de inúmeros fatores que não serão aqui relacionados. Todavia, é importante destacar que todos esses medicamentos são de uso recente e não há dados conclusivos para definir a sua segurança. A maioria dos estudos ainda é resultado de análise de exposições em modelos animais e o valor preditivo de risco de efeito adverso para os humanos não está estabelecido.

\section{Efeitos das drogas na gravidez}

O Centro Regulador de Alimentos e Drogas dos Estados Unidos da América (FDA - Food and Drug Administration) elaborou recomendações acerca do uso de anti-retrovirais, segundo os conhecimentos atuais de dados clínicos e pré-clínicos, sendo em sua maioria baseados em estudos em animais. Classifica as categorias das drogas na gravidez em: A- dados baseados em estudos adequados, controlados e que não demonstraram risco para o feto durante o primeiro trimestre de gravidez e não há risco nos trimestres subseqüentes; B- estudos em animais durante a reprodução que não revelaram risco para o feto, mas não há estudos controlados em humanos; C- a segurança na gravidez humana não foi determinada. Os estudos em animais ou foram positivos para risco fetal ou não foram realizados e a droga não deve ser utilizada a menos que os beneficios potenciais superem os riscos para o feto; D- há evidência documentada de risco fetal humano baseada em registro de efeitos adversos em investigação clínica ou relatos durante seu uso na prática clínica, mas os beneficios potenciais para as gestantes superam quaisquer riscos; X- estudos em animais ou relatos de reações adversas indicam que os riscos associados ao uso da droga na gestação claramente superam quaisquer beneficios possiveis.

A classificação atual das drogas anti-retrovirais pode ser observada no Quadro 1. A maioria das as está classificada nas categorias B ou C, nenhuma na categoria $\mathrm{A}$, sendo que o efavirenz recentemente passou a ser classificado na categoria D. Entretanto, muitos dos estudos de observação em longo prazo não foram completados. Mais informações podem ser obtidas no endereço eletrônico: www.aidsinfo.nih.gov, em perinatal guidelines (consultar guidelines e supplements) ou no www.apregistry.com construído para receber registros de efeitos adversos dos anti-retrovirais na gravidez. Neste registro com dados até julho de 2005 é destacado que a prevalência de defeitos em nascidos vivos expostos no primeiro trimestre de gravidez variou entre 2 e $4 \%$ e que é mais elevada com o uso de didanosina. Observe-se que a taxa de malformações da população geral nos Estados Unidos é de $3 \%{ }^{2}$.

Podemos resumir as seguintes informações sobre as drogas e seus efeitos na gestação e feto:

1) Zidovudina é aquela sobre a qual se têm mais informações devido a seu amplo uso há mais de 10 anos, sendo considerada segura. O efeito adverso observado no recém-nascido em curto prazo é a anemia, em geral de intensidade leve que resolve espontaneamente na maioria das ocasiões. Não demanda transfusões sanguineas, em geral não alcança niveis de hemoglobina inferiores a $10 \mathrm{~g} \%$ e reverte após a suspensão da droga. Porém, esse efeito do AZT antes reconhecido como transitório e aparentemente mais restrito à série vermelha e aos neutrófilos hoje tem sido mais estudado. Em estudo longitudinal de crianças expostas ao AZT isolado ou combinado, acompanhadas até 18 meses de vida, verificou-se que o efeito é moderado e mais prolongado acometendo todas as séries hematológicas: neutrófilos, plaquetas e linfócitos, incluindo os linfócitos TCD4+ e TCD8+ ${ }^{3}$. Em dados mais recentes do Estudo Colaborativo Europeu, com o acompanhamento de cerca de 1500 crianças por até 8 anos, verificou-se contagem de neutrófilos significantemente menor em crianças expostas a profilaxia antiretroviral intra-útero (a maioria com o uso de zidovudina em monoterapia) comparada com 
Quadro 1 - Dados clínicos e pré-clínicos relevantes acerca do uso de drogas anti-retrovirais durante a gestação.

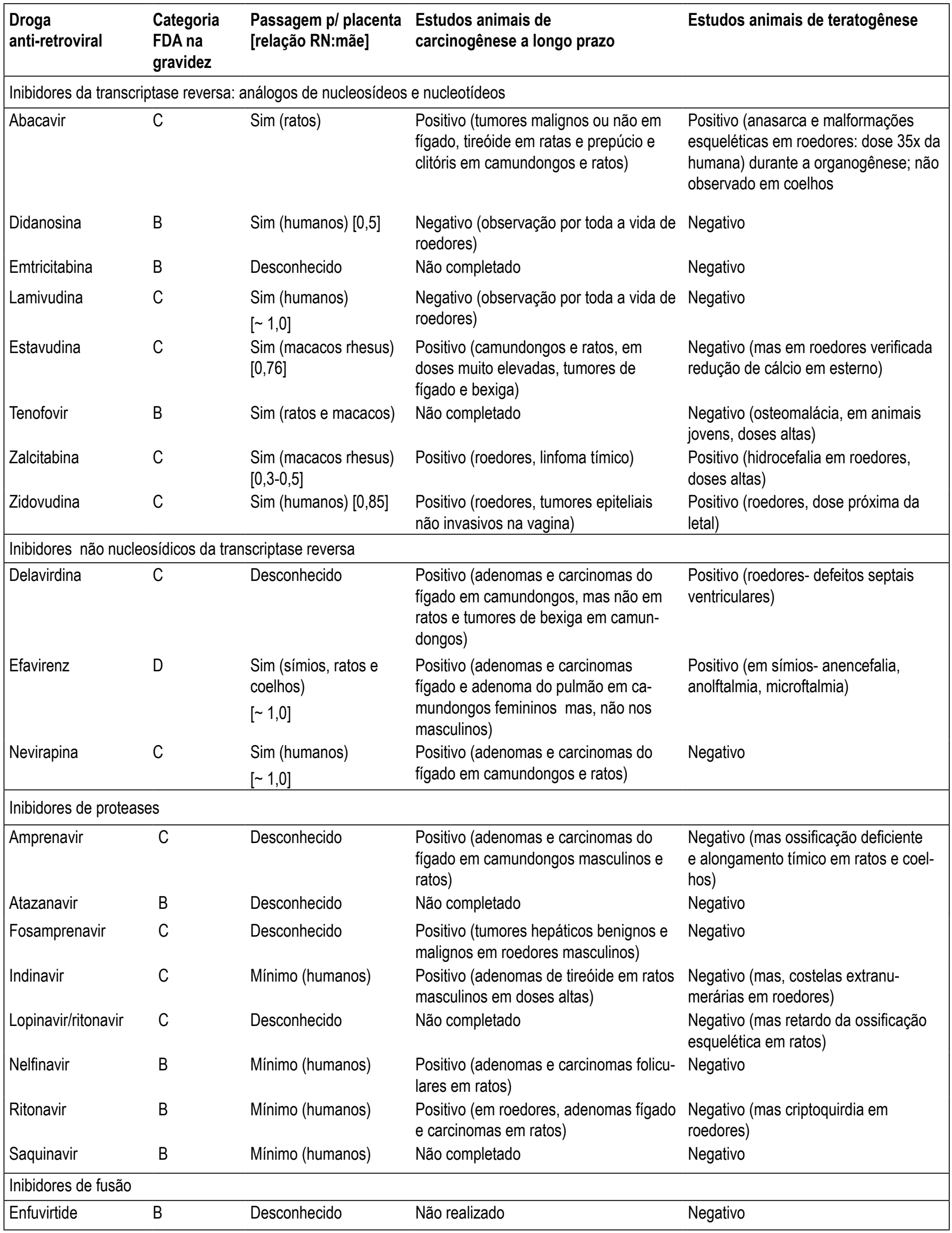


crianças não expostas, e os autores consideram que a avaliação em longo prazo das alterações hematológicas deva ser continuada ${ }^{4}$. Até o momento não houve aumento da incidência de malformações congênitas nas crianças expostas quando comparada com a da população geral $^{2}$. Também não foi identificado impacto desfavorável no crescimento e desenvolvimento. Com relação ao potencial carcinogênico, há dois estudos realizados em camundongos com resultados divergentes: no primeiro, o uso de doses elevadas, aproximadamente 25 a 50 vezes a dose terapêutica para os humanos, esteve associada com aumento de tumores de pulmão, figado e trato genital feminino, e no segundo, utilizando doses menores, cerca de três vezes a dose terapêutica para humanos, não foi observado aumento do surgimento de tumores. O acompanhamento de mais de 700 crianças com exposição intra-uterina ao AZT por período superior a seis anos não observou a ocorrência de doenças malignas. Desse modo, depois da avaliação aprofundada dos dados disponiveis concluiu-se que os beneficios superam os riscos teóricos, porém, recomenda-se o seguimento das crianças até o final da adolescência com exames clínicos periódicos (anuais).

2) Efavirenz - em estudos em primatas, o uso da droga na gestação, em doses comparáveis às dos humanos, esteve associada com defeitos congênitos considerados graves: anencefalia, anoftalmia, microcefalia e defeitos do palato, sugerindo que seu uso deve ser evitado na gravidez. Existem relatos de cinco fetos/recém-nascidos humanos com defeitos do tubo neural (quatro com meningomielocele e um com a sindrome de Dandi Walker) gerados por mulheres que receberam efavirenz no início da gestação ${ }^{5-8}$.

3) Nelfinavir - dois estudos europeus faziam referência a um aumento de risco de hemangiomas em crianças expostas às drogas antiretrovirais durante a gestação $0^{9,10}$. De Santis et al. ${ }^{11}$ também relataram aumento de risco de hemangiomas $(>5 \mathrm{~cm}$ ) de $4,2 \%$ comparado com $0,3 \%$ no grupo controle, em crianças cujas mães receberam zidovudina, lamivudina e nelfinavir durante a gravidez. Esses autores chamam a atenção para essa possivel associação e estimulam os pesquisadores a relatarem suas experiências antes que se possa ter uma conclusão mais definitiva.

4) Outras drogas - além dos anti-retrovirais, outras drogas usadas pela mãe podem também ser teratogênicas. O fluconazol foi associado com presença de anomalias crânio-faciais, esqueléticas e cardiacas nas crianças expostas intra-útero ${ }^{12,13}$.

5) Prematuridade - Foram observadas taxas mais elevadas de partos prematuros entre gestantes que estiveram sob uso de terapia anti-retroviral combinada ${ }^{9,14}$. No entanto, em revisões sistemáticas isso não foi observado ${ }^{15,16}$. De qualquer forma permanece a preocupação com tal ocorrência, que aumenta a morbidade e mortalidade perinatais.

6) Toxicidade mitocondrial - O aumento transitório do lactato sérico tem sido observado em crianças expostas aos inibidores nucleosídicos da transcriptase reversa, mas não se sabe o seu significado clínico ${ }^{17,18}$. Há o relato de crianças expostas ao HIV que não se infectaram e foram expostas intra-útero a inibidores nucleosídicos da transcriptase reversa (AZT e/ou lamivudina) que apresentaram evidência clínica e laboratorial de disfunção mitocondrial. Isso ocorreu em oito de 1754 crianças, sendo que duas delas faleceram com manifestações neurológicas graves; três apresentaram sintomas leves a moderados, incluindo miocardiopatia transitória, e três restantes tiveram niveis elevados de lactato sem repercussão clínica ${ }^{19}$. Com dados acumulados subseqüentes identificaram-se mais quatro crianças, totalizando doze crianças com disfunção mitocondrial, o que projeta incidência do agravo de $0,26 \%$ aos 18 meses de idade ${ }^{20}$. Também há o relato de um caso de acidose láctica grave em recém-nascido que se resolveu após a suspensão do $\mathrm{AZT}^{21}$ e de uma criança com macrossomia, cardiomiopatia e hepatomegalia, hipoglicemia, aumento das transaminases e de lactato que faleceu no período neonatal, nascida de mãe sob tratamento com terapia anti-retroviral combinada. Os pesquisadores não puderam explicar os achados pela asfixia neonatal, anormalidade cromossômica ou qualquer defeito metabólico conhecido, sugerindo haver relação entre os achados na criança e o uso de medicação pela mãe ${ }^{22}$. Poirier et al. ${ }^{23}$ encontraram quantidade menor de DNA mitocondrial em grupos de crianças expostas comparadas com aquelas não expostas ao AZT. Apesar da disfunção mitocondrial ainda ser considerada questão controversa descrita por alguns grupos, mas não observada por outros, recomenda-se o seguimento em longo prazo das crianças expostas aos anti-retrovirais em diferentes artigos e consensos de diversos países, por meio de acompanhamento clínico e avaliação do desenvolvimento neuropsicomotor ${ }^{24-28}$. 
7) Convulsões febris - No estudo de coorte francês, observou-se aumento na taxa de convulsões febris precoces em crianças não infectadas expostas a anti-retrovirais, cujo risco cumulativo foi de $11 / 1000$ crianças $(1,1 \%)$, comparado com risco de 4,1/1000 (0,4\%) nas crianças do grupo sem exposição aos anti-retrovirais ${ }^{29}$.

8) Alterações cardíacas - Há preocupação especial com o risco de manifestações cardiovasculares, desde miocardiopatia assintomática até quadros de grave insuficiência cardíaca. Nos estudos que relataram disfunção mitocondrial, algumas das crianças também apresentaram miocardiopatia. Lipshultz et al. ${ }^{30}$ avaliando prospectivamente cerca de 400 crianças nascidas de mães infectadas pelo HIV, expostas ou não a anti-retrovirais, não encontraram evidência de alterações cardiacas relacionadas à exposição intra-uterina. Adicionalmente, em outro estudo verificou-se que crianças nascidas de mães infectadas, independente de terem ou não sido infectadas, apresentam anormalidades cardiovasculares persistentes com função ventricular esquerda e cardíaca pior do que crianças do grupo controle, sugerindo o acompanhamento em longo prazo das crianças não infectadas ${ }^{31}$.

9) Redução dos níveis séricos de insulina no recém-nascido - pesquisadores brasileiros encontraram niveis de insulina inferiores em recém-nascidos expostos à terapia anti-retroviral combinada intra-útero comparados a um grupo controle $(p<0,05)$, embora não tenham verificado diferença na glicemia neonatal ${ }^{32}$. Os autores julgam que tais resultados podem ser explicados devido à observação de que a maioria dos anti-retrovirais diminui a tolerância à glicose tanto pela secreção e/ou ação diminuída da insulina ou mesmo pelo efeito tóxico direto desses agentes sobre as células beta-pancreáticas ${ }^{33}$. Sugerem ainda que o uso dos inibidores de protease tem sido relacionado com o surgimento de intolerância à glicose e casos de diabetes ${ }^{34}$.

\section{Conclusão e Recomendações de acompanhamento}

\section{das crianças expostas}

Baseados nestes conhecimentos atuais, considera-se que o beneficio na acentuada redução da transmissão vertical do HIV com o uso de drogas anti-retrovirais ultrapassa os riscos eventuais dos efeitos adversos. Entretanto, até que experiência de longo prazo se acumule, é necessário acompanhamento clínico especializado e rigoroso das crianças expostas. Os vários consensos e revisões sugerem que este seja feito mais amiúde ao longo do primeiro ano e depois anualmente até o final da adolescência. A operacionalização das consultas em longo prazo deve ser adaptada às condições de cada serviço ou região.

A anamnese deve ser minuciosa acerca das condições habituais de vida da criança, alimentação, sono, comportamento e intercorrências infecciosas ou não. Deve-se averiguar a presença de sinais e sintomas sugestivos de toxicidade mitocondrial, variáveis e nem sempre específicos, podendo incluir nos casos mais graves manifestações neurológicas como encefalopatia, convulsões afebris e retardo do desenvolvimento; sintomas cardiacos devidos a miocardiopatia e disfunção de ventrículo esquerdo; sintomas gastrintestinais atribuiveis à hepatite (esteatose hepática), além de outras síndromes clínicas como miopatia, retinopatia, pancreatite e acidose láctica ${ }^{23-25,35-38}$.

O exame físico deve conter: peso; comprimento ou estatura; perímetro cefálico (até os 4 anos de idade); medida de pressão arterial (em pelo menos uma das visitas deve ser feita nos 4 membros), freqüência cardiaca e respiratória; descrição e localização de lesões de pele (inclusive de hemangiomas, medindo-se o seu tamanho e evolução); descrição de alterações estruturais ou anomalias presentes; presença ou não de gânglios (descrição das cadeias envolvidas, tamanho, aspecto e consistência dos gânglios). Relatar outras alterações com detalhes e incluir sempre a avaliação do desenvolvimento neuropsicomotor.

A avaliação laboratorial deve incluir o hemograma completo, recomendado em todas as consultas do primeiro ano de vida e anualmente, devido aos riscos do HIV, AZT e da terapia combinada sobre as séries hematológicas. Outros exames são as provas de função hepática e glicemia, buscando acompanhar o risco potencial de alterações metabólicas relatadas nas crianças expostas ${ }^{39}$. No consenso britânico também são incluídas avaliações periódicas de uréia e eletrólitos e recomendam a monitorização de $\mathrm{pH}$ e ácido láctico em crianças sintomáticas diante de suspeita de toxicidade mitocondrial não sendo indicada em crianças com boa evolução clínica ${ }^{40}$. No Brasil, essa recomendação pode ser encontrada no consenso para crianças expostas e infectadas do Ministério da Saúde (www.aids.gov.br).

Consultas com outros especialistas como neuropediatra ou cardiologista infantil poderão ser necessárias e presença de dados de história e/ou 
alterações no exame físico ou de achados laboratoriais ou de imagem sugestivos da presença de toxicidade mitocondrial.

Sugere-se utilizar a Caderneta de Saúde da Criança do Ministério da Saúde (disponivel em: htpp://dtr2001.saude.gov.br/bvs/publicacoes / menina_final.pdf e menino_final.pdf) ou algo similar, mantendo o registro atualizado da evolução clínica com a mãe ou responsável para permitir acesso a informação inclusive por outros profissionais de saúde que realizem qualquer atendimento fora do acompanhamento rotineiro da sua condição de criança exposta ao HIV e anti-retrovirais intra-útero.

\section{Referências}

1. Connor EM, Sperling RS, Gelber R, Kiselev P, Scott G, O'Sullivan MJ, et al. Reduction of maternal-infant transmission of human immunodeficiency virus type 1 with zidovudine treatment. Pediatric AIDS Clinical Trials Group Protocol 076 Study Group. N Engl J Med. 1994;331(18):1173-80.

2. Correa-Villasenor A, Cragan J, Kucik J, O’Leary L, Siffel C, Williams L. The Metropolitan Atlanta Congenital Defects Program: 35 years of birth defects surveillance at the Centers for Disease Control and Prevention. Birth Defects Res A Clin Mol Teratol. 2003;67(9):617-24.

3. Le Chenadec J, Mayaux MJ, Guihenneuc-Jouyaux C, Blanche S; Enquete Perinatale Francaise Study Group. Perinatal antiretroviral treatment and hematopoiesis in HIV-uninfected infants. AIDS. 2003;17(14):2053-61.

4. European Collaborative Study. Levels and patterns of neutrophil counts over the first 8 years of life in children of life of HIV-1-infected mothers. AIDS. 2004;18(15):2009-17.

5. Richardson MP, Osrin D, Donaghy S, Brown NA, Hay P, Sharland M. Spinal malformations in the fetuses of HIV infected women receiving combination antiretroviral therapy and co-trimoxazole. Eur J Obstet Gynecol Reprod Biol. 2000; 93(2):215-7.

6. Fundarò C, Genovese O, Rendeli C, Tamburrini E, Salvaggio E. Myelomeningocele in a child with intrauterine exposure to efavirenz. AIDS. 2002;16(2):299-300.

7. De Santis M, Carducci B, De Santis L, Cavaliere AF, Straface G. Periconceptional exposure to efavirenz and neural tube defects. Arch Intern Med. 2002; $162(3): 355$.

8. Saitoh A, Hull AD, Franklin P, Spector SA. Myelomeningocele in an infant with intrauterine exposure to efavirenz. J Perinatol. 2005;25(8):555-6.

9. Lorenzi P, Spicher VM, Laubereau B, Hirschel B, Kind C, Rudin C, et al. Antiretroviral therapies in pregnancy: maternal, fetal and neonatal effects. Swiss HIV Cohort Study, the Swiss Collaborative HIV and Pregnancy Study, and the Swiss Neonatal HIV Study. AIDS. 1998;12(18):F241-7.

10.Simon T, Funke AM, Hero B, Reiser-Hartwig S, Fuhrmann U.[Efficiency and side effects of antiretroviral treatment of HIV infected pregnant women]. Zentralbl Gynakol. 2002; 124(8-9):41 3-7. German.

11.De Santis M, Cavaliere AF, Caruso A, Villa P, Tamburini E, Cauda R, et al. Hemangiomas and other congenital malformations in infants exposed to antiretroviral therapy in utero. JAMA. 2004;291(3):1961-2.

12.Pursley TJ, Blomquist IK, Abraham J, Andersen HF, Bartley JA. Fluconazole-induced congenital anomalies in three infants. Clin Infect Dis. 1996;22(2): 336-40.

13. Jick SS. Pregnancy outcomes after maternal exposure to fluconazole. Pharmacotherapy. 1999;19(2):221-2.

14.European Collaborative Study; Swiss Mother and Child HIV Cohort Study. Combination antiretroviral therapy and duration of pregnancy. AIDS. 2000;14(18):2913-20.

15.Brocklehurst P, French R. The association between maternal HIV infection and perinatal outcome: a systematic review of the literature and meta-analysis. Br J Obstet Gynaecol. 1998;105(8):836-48.

16.Tuomala RE, Shapiro DE, Mofenson LM, Bryson Y, Culnane M, Hughes MD, et al. Antiretroviral therapy during pregnancy and the risk of an adverse outcome. N Engl J Med. 2002;346(24):1863-70.

17. Giaquinto C, De Romeo A, Giacomet V, Rampon O, Ruga E, Burlina A, et al. Lactic acid levels in children perinatally treated with antiretroviral agents to prevent HIV transmission. AIDS. 2001; 15(8): 1074-5.

18.Alimenti A, Burdge DR, Ogilve GS, Money DM, Forbes JC. Lactic acidemia in human immunodeficiency virusuninfected infants exposed to perinatal antiretroviral therapy. Pediatr Infect Dis J. 2003;22(9):782-9.

19.Blanche S, Tardieu M, Rustin P, Slama A, Barret B, Firtion $\mathrm{G}$, et al. Persistent mitochondrial dysfunction and perinatal exposure to antiretroviral nucleoside analogues. Lancet. 1999;354(9184):1084-9.

20.Barret B, Tardieu M, Rustin P, Lacroix C, Chabrol B, Desguerre I, et al. Persistent mitochondrial dysfunction in HIV-1 exposed but uninfected infants: clinical screening in a large prospective cohort. AIDS. 2003; 17(12):1769-85.

21.Scalfaro P, Chesaux JJ, Buchwalder PA, Biollaz J, Micheli JL. Severe transient neonatal lactic acidosis during prophylactic zidovudine treatment. Intensive Care Med. 1998; 24(3):247-50.

22.Godfried $\mathrm{MH}$, Boer K, Beuger S, Scherpbier HJ, Kuijpers TW. A neonate with macrosomia, 
cardiomyopathy and hepatomegaly born to an HIVinfected mother. Eur J Pediatr. 2005, 164(3):190-2.

23.Poirier MC, Divi RL, Al-Harthi L, Olivero OA, Nguyen $\mathrm{V}$, Walker B, et al. Long-term mitochondrial toxicity in HIV-uninfected infants born to HIV-infected mothers. J Acquir Immune Defic Syndr. 2003;33(2):175-83.

24.King SM; American Academy of Pediatrics Committee on Pediatric AIDS; American Academy of Pediatrics Infectious Diseases and Immunization Committee. Evaluation and treatment of the human immunodeficiency virus-1 - exposed infant. Pediatrics. 2004; $114(2): 497-505$.

25.El Beitune P, Duarte G, Quintana SM, Figueiró-Filho E, Marcolin AC, Abduch R. Antiretroviral therapy during pregnancy and early neonatal life: Consequences for HIV- exposed, uninfected children. Braz J Infect Dis. 2004;8(2):140-50.

26. Shetty AK. Perinatally acquired HIV-1 infection: prevention and evaluation of HIV-exposed infants. Semin Pediatr Infect Dis. 2005;16(4):282-95.

27. Foster C, Lyall H. Current Guidelines for the management of UK infants born to HIV-1 infected mothers. Early Hum Dev. 2005; 81(1):103-10.

28. Thorne C, Newell ML. The safety of antiretroviral drugs in pregnancy. Expert Opin Drug Saf. 2005;4(2): 323-35.

29.Landreau-Mascaro A, Barret B, Mayaux MJ, Tardieu M, Blanche S; French Perinatal Cohort Study Group. Risk of early febrile seizure with perinatal exposure to nucleoside analogues. Lancet. 2002; 359(9306): 583-4.

30.Lipshultz SE, Easley KA, Orav EJ, Kaplan S, Starc TJ, Bricker JT, et al. Absence of cardiac toxicity of zidovudine in infants. Pediatric Pulmonary and Cardiac Complications of Vertically Transmitted HIV Infection Study Group. N Engl J Med. 2000;343(11 ):759-66.

31.Lipshultz SE, Easley KA, Orav EJ, Kaplan S, Starc TJ, Bricker JT, et al. Cardiovascular status of infants and children of women infected with HIV-1 (P2C2HIV): A cohort study. Lancet. 2002; 360(9330):368-73.
32.El Beitune P, Duarte G, Foss MC, Montenegro RM Jr, Quintana SM, Figueiró- Filho EA, et al. Effect of maternal use of antiretroviral agents on serum insulin levels of the newborn infant. Diabetes Care. 2005,28(4):856-9.

33.Figueiró-Filho EA, El Beitune P, Rudge MVC, Quintana SM, Marcolin AC, Duarte G. Effects of antiretroviral drugs on glucide metabolism and pancreatic Langerhans's cells of pregnant Wistar rats. Rev Bras Ginecol Obstet. 2004,26(5):369-75.

34. US Public Health Service Task Force. Recommendations for the use of antiretroviral drugs in pregnant HIV-1 infected women for maternal health and interventions to reduce perinatal HIV-1 transmission in the United States. Supplement: Safety and Toxicity of Individual Antiretroviral Agents in Pregnancy. Rockville: National Institute of Health; 2005 [cited 2005 Nov 17]. Available from: http://www.aidsinfo.nih.gov

35.Johns DR. Seminars in medicine of the Beth Israel Hospital, Boston. Mitochondrial DNA and disease. N Engl J Med. 1995;333(10):638-44.

36.Brinkman K, ter-Hofstede HJM, Burger DM, Smeitink JA, Koopmans PP. Adverse effects of reverse transcriptase inhibitors: Mitochondrial toxicity as commom pathway. AIDS. 1998;12(14):1735-44.

37.Culnane M, Fowler M, Lee SS, McSherry G, Brady M, O'Donnell K, et al. Lack of long-term effects of in utero exposure to zidovudine among uninfected children born to HIV-infected women. Pediatric AIDS Clinical Trials Group Protocol 219/076 Teams. JAMA. 1999;281(2):151-7.

38.KristAH, Crawford-Faucher A. Management of newborns exposed to maternal HIV infection. Am Fam Physician. 2002; 65(10): 2049-56.

39. Taha TE, Kumwenda N, Gibbons A, Hoover D, Lema V, Fiscus S, et al. Effect of antiretroviral prophylaxis on hepatic and hematological parameters of African infants. AIDS. 2002;16(6):851-8.

40. British HIV Association. Guidelines for the management of HIV infection in pregnant women and the prevention of mother-to-child transmission of HIV. March 2005 [cited 2005 Dec 19]. Available from: http://www.bhiva.org 